\title{
In vitro antifungal activity of essential oils extracted from plants against fluconazole-susceptible and resistant Candida albicans
}

\section{Farzad Katiraee, ${ }^{1,}{ }^{*}$ Somayeh Ahmadi Afshar, ${ }^{1}$ Seyedeh Faezeh Rahimi Pirmahalleh, ${ }^{1}$ and $\underline{\text { Hojjatolah Shokri }}{ }^{2}$}

1Department of Pathobiology, Faculty of Veterinary Medicine, University of Tabriz, Tabriz, Iran

2Department of Pathobiology, Faculty of Veterinary Medicine, Amol University of Special Modern Technologies, Amol, Iran

\begin{abstract}
Background and Purpose: Candida albicans is the most common cause of candidal infections. Various studies have shown drug resistance among $C$. albicans isolates; thus, it is necessary to discover replacement treatments for Candida infections. In this study, we aimed to compare the effects of different essential oils against azoles-resistant and azolessusceptible isolates.
\end{abstract}

Materials and Methods: Twenty fluconazole-resistant and 20 susceptible $C$. albicans isolates obtained from oral, vaginal, and cutaneous tissues of patients with candidiasis were evaluated. The efficacy and minimum inhibitory concentrations (MICs) of Zataria multiflora, Geranium herbarum, Lavendula officinalis, Cuminum, cyminum, Allium heamanthoides, and Artemisia sieberi essential oils against $C$. albicans were determined on the basis of a reference method for broth microdilution susceptibility testing of yeasts as suggested by Clinical and Laboratory Standards Institute (CLSI, M27-S4). After inoculation, incubation, and subculturation, the MICs were determined through comparison with the control.

\begin{tabular}{|c|c|c|c|c|c|}
\hline Essences & Source of isolation(n) & Means & Min & Max & Significance \\
\hline \multirow{3}{*}{$\begin{array}{l}\text { Zataria } \\
\text { multiflora }\end{array}$} & Oral(15) & 0.142 & 0.1 & 0.25 & \multirow[t]{3}{*}{ No Significan } \\
\hline & Vaginal(15) & 0.165 & 0.125 & 0.25 & \\
\hline & Cutaneous(10) & 0.17 & 0.125 & 0.25 & \\
\hline \multirow{3}{*}{$\begin{array}{l}\text { Geranium } \\
\text { herbarum }\end{array}$} & Oral(15) & 1.0 & 0.625 & 1.66 & \multirow[t]{3}{*}{ No Significant } \\
\hline & Vaginal(15) & 0.904 & 0.625 & 1.25 & \\
\hline & Cutaneous(10) & 0.883 & 0.625 & 1.25 & \\
\hline \multirow{3}{*}{$\begin{array}{l}\text { Artemisia } \\
\text { sieberi }\end{array}$} & Oral(15) & 1.291 & 1.0 & 1.66 & \multirow[t]{3}{*}{ No Significant } \\
\hline & Vaginal(15) & 1.173 & 0.833 & 1.66 & \\
\hline & Cutaneous(10) & 1.174 & 0.833 & 2.0 & \\
\hline \multirow{3}{*}{$\begin{array}{l}\text { Allium } \\
\text { heamanthoides }\end{array}$} & Oral(15) & 0.14 & 0.1 & 0.25 & \multirow[t]{3}{*}{ No Significant } \\
\hline & Vaginal(15) & 0.165 & 0.1 & 0.25 & \\
\hline & Cutaneous(10) & 0.17 & 0.125 & 0.25 & \\
\hline \multirow{3}{*}{$\begin{array}{l}\text { Cumminum } \\
\text { cyminum }\end{array}$} & Oral(15) & 3.05 & 2.0 & 4.0 & \multirow[t]{3}{*}{ No Significant } \\
\hline & Vaginal(15) & 3.2 & 2.0 & 4.0 & \\
\hline & Cutaneous (10) & 3.15 & 2.0 & 4.0 & \\
\hline \multirow{3}{*}{$\begin{array}{l}\text { Lavendula } \\
\text { officinalis }\end{array}$} & Oral(15) & 2.55 & 1.5 & 3.0 & \multirow[t]{3}{*}{ No Significar } \\
\hline & Vaginal(15) & 2.6 & 1.5 & 3.0 & \\
\hline & Cutaneous(10) & 2.35 & 1.5 & 3.5 & \\
\hline
\end{tabular}

Table 1

The obtained minimum inhibitory concentrations for Zataria multiflora, Geranium herbarum, Artemisia sieberi, Allium, Cumminum cyminum, and Lavendula officinalis based on source of isolation
Results: The obtained MICs for Zataria multiflora, Geranium herbarum, Artemisia sieberi, Allium heamanthoides, Cumminum cyminum, and Lavendula officinalis were 0.1-0.25 $\mathrm{\mu l} / \mathrm{ml}$ (mean: $0.155 \mu \mathrm{l} / \mathrm{ml}$ ), 0.625-1.66 $\mathrm{ll} / \mathrm{ml}$ (mean: $0.93 \mu \mathrm{l} / \mathrm{ml}$ ) 0.833-2.0 $\mu \mathrm{l} / \mathrm{ml}$ (mean: $1.21 \mu \mathrm{l} / \mathrm{ml}$ ), 0.1-0.25 $\mu \mathrm{l} / \mathrm{ml}$ (mean: $0.155 \mu \mathrm{l} / \mathrm{ml}$ ), $2-4 \mu \mathrm{l} / \mathrm{ml}$ (mean: $3.1 \mu \mathrm{l} / \mathrm{ml}$ ), and 1.5-3.0 $\mu \mathrm{l} / \mathrm{ml}$ (mean: $2.4 \mu \mathrm{l} / \mathrm{ml}$ ), respectively. The results showed that Zataria multiflora and Allium heamanthoides essential oils were more efficient than other essential oils against Candida species. There were no significant differences between various Candida strains in terms of susceptibility to the essential oils. In addition, there were no significant differences in the MICs of these essential oils against the azoles-resistant and azoles-susceptible isolates.

Conclusion: In this study, the anti-Candida effects of six essential oils against both azoles-resistant and azolessusceptible isolates were similar. Given the documented resistance of different Candida species to synthetic and chemical antifungals, these essential oils are effective replacement treatments for cutaneous and mucosal Candida infections, especially in resistant or recurrent cases.

Keywords: Antifungal resistance, Candida albicans, Essential oils

\begin{tabular}{|c|c|c|c|c|c|}
\hline Essences & $\begin{array}{l}\text { Susceptibility } \quad \text { to } \\
\text { fluconazole }(n)\end{array}$ & Means & Min & Max & Sign \\
\hline \multirow[t]{2}{*}{ Zataria multiflora } & Resistance(20) & 0.161 & 0.1 & 0.25 & \multirow[t]{2}{*}{ NS } \\
\hline & Susceptible(20) & 0.156 & 0.1 & 0.25 & \\
\hline \multirow{2}{*}{$\begin{array}{l}\text { Geranium } \\
\text { herbarum }\end{array}$} & Resistance(20) & 0.874 & 0.625 & 1.25 & \multirow[t]{2}{*}{ NS } \\
\hline & Susceptible(20) & 0.985 & 0.625 & 1.66 & \\
\hline \multirow[t]{2}{*}{ Artemisia sieberi } & Resistance(20) & 1.21 & 0.833 & 2.0 & \multirow[t]{2}{*}{ NS } \\
\hline & Susceptible(20) & 1.215 & 0.833 & 1.66 & \\
\hline \multirow{2}{*}{$\begin{array}{l}\text { Allium } \\
\text { heamanthoides }\end{array}$} & Resistance(20) & 0.165 & 0.1 & 0.25 & \multirow[t]{2}{*}{ NS } \\
\hline & Susceptible(20) & 0.152 & 0.1 & 0.25 & \\
\hline \multirow{2}{*}{$\begin{array}{l}\text { Cumminum } \\
\text { cyminum }\end{array}$} & Resistance(20) & 3.033 & 2.0 & 4.0 & \multirow[t]{2}{*}{ NS } \\
\hline & Susceptible(20) & 3.233 & 2.0 & 4.0 & \\
\hline \multirow{2}{*}{$\begin{array}{l}\text { Lavendula } \\
\text { officinalis }\end{array}$} & Resistance(20) & 2.2 & 1.5 & 3.0 & \multirow[t]{2}{*}{ NS } \\
\hline & Susceptible(20) & 2.6 & 1.5 & 3.5 & \\
\hline \multirow[t]{2}{*}{ Total } & Resistance(20) & 1.274 & 0.1 & 4.0 & \multirow[t]{2}{*}{ NS } \\
\hline & Susceptible(20) & 1.39 & 0.1 & 4.0 & \\
\hline
\end{tabular}

Table 2

The obtained minimum inhibitory concentrations for Zataria multiflora, Geranium herbarum, Artemisia sieberi, Allium, Cumminum cyminum, and Lavendula officinalis based on susceptibility of strains to fluconazole 